\title{
Hyperthermic intraperitoneal perfusion chemotherapy and response evaluation in patients with gastric cancer and malignant ascites
}

\author{
XUEFENG NI $^{1 *}$, PING WU ${ }^{2 *}$, JUN WU $^{1}$, MEI JI ${ }^{1}$, BO TIAN ${ }^{3}$, ZHENXING JIANG $^{4}$, \\ YUE SUN $^{5}$, XIAOXIAO XING ${ }^{5}$, JINGTING JIANG ${ }^{1}$ and CHANGPING WU ${ }^{1}$ \\ Departments of ${ }^{1}$ Oncology, ${ }^{2}$ Pharmacology, ${ }^{3}$ Pathology, ${ }^{4}$ Radiology and ${ }^{5}$ Ultrasonography, \\ The Third Affiliated Hospital, Soochow University, Changzhou, Jiangsu 213003, P.R. China
}

Received September 15, 2015; Accepted March 3, 2017

DOI: $10.3892 / 01.2017 .6342$

\begin{abstract}
The present study describes the use of bidirectional chemotherapy in the treatment of patients with gastric cancer and peritoneal carcinomatosis (PC), using newly developed response criteria for the treatment of malignant ascites. In addition, the association between effusion response and survival was analyzed. Between June 2010 and May 2014, patients affected by malignant ascites secondary to unresectable PC of gastric origin were treated with a combination of systemic and loco-regional chemotherapy. Cisplatin $\left(75 \mathrm{mg} / \mathrm{m}^{2}\right)$ at an inflow temperature of $43^{\circ} \mathrm{C}$ was infused intraperitoneally, and docetaxel $\left(75 \mathrm{mg} / \mathrm{m}^{2}\right)$ was infused simultaneously via a peripheral vein, on day 1 every 3 weeks. The primary endpoint was overall survival rate, and the secondary endpoint was efficacy against malignant ascites using new response criteria. In total, 41 patients were enrolled, the majority of whom received 6 cycles of intraperitoneal chemotherapy in combination with hyperthermia. The majority of patients exhibited clinical regression of ascites and relief of associated symptoms. Malignant ascites disappeared [complete response (CR)] or decreased by $50 \%$ [partial response (PR)] in $73.2 \%$ of patients. No mortalities associated with the procedures occurred. The median survival time was 8.6 months, and the 1-year survival rate was $24.4 \%$. As these new response criteria for the treatment of malignant ascites were found to be feasible, bidirectional chemotherapy may be the preferred strategy for the treatment of gastric cancer with PC. The CR, PR and non-PR groups showed significant differences in overall survival, indicating
\end{abstract}

Correspondence to: Dr Changping Wu, Department of Oncology, The Third Affiliated Hospital, Soochow University, 185 Juqian Road, Changzhou, Jiangsu 213003, P.R. China

E-mail: wcpzlk@163.com

${ }^{*}$ Contributed equally

Key words: gastric cancer, malignant ascites, hyperthermic intraperitoneal perfusion chemotherapy, response evaluation that decreased effusion was associated with improved patient survival.

\section{Introduction}

Although the incidence of gastric cancer has decreased worldwide, particularly in Western countries, it remains the fourth most common type of cancer and the second-most common cause of cancer-associated mortality worldwide $(1,2)$. Peritoneal carcinomatosis (PC) is the most frequent mode of recurrence and is responsible for $\sim 60 \%$ of gastric cancer-associated mortalities (3). Patients with gastric cancer exhibiting PC are considered not to be curable and are usually treated with systemic chemotherapy without surgical resection $(4,5)$. Patients with PC and ascites have very poor prognoses, with a median survival of 3-6 months, and there are no long-term survivors $(4,5)$. PC is a common event in the natural history of gastrointestinal tract cancers, with a high 6-month mortality rate (6). Malignant ascites, one of the most frequent comorbid conditions complicating PC (7), may severely affect patient quality of life, and its symptoms may be particularly painful and life threatening $(7,8)$.

PC from gastrointestinal cancers has been considered an incurable condition, for which the role of surgical intervention is limited (9). Systemic chemotherapy is the treatment of choice, with significant survival benefits compared with best-supportive care, but even systemic chemotherapy is relatively ineffective against PC due to the blood-peritoneal barrier, which consists of a monolayer of mesothelial cells and submesothelial connective tissue $\sim 90 \mu \mathrm{m}$ thick between the basement membrane and vasculature (10).

Intraperitoneal (IP) chemotherapy is designed to increase the dose and exposure time of intra-abdominal cancer cells to anticancer drugs, while minimizing systemic toxic effects $(11,12)$. Prolonged retention in the peritoneal cavity and clearance from the systemic circulation are regarded as key attributes for IP chemotherapy drugs $(11,12)$. Heat has been exhibited to be synergistic with the antitumoral effects of mitomycin C, cisplatin (CDDP) and oxaliplatin $(13,14)$.

Drugs regarded as ideal for IP administration are those that maintain a high concentration in the peritoneum, exhibit high 
penetration into the tumors and have low systemic concentrations; CDDP and carboplatin have been shown to penetrate 1-2 mm from the surface of PC nodules (15). These two drugs are easily absorbed from the peritoneal cavity and are suitable for IP chemotherapy (15). CDDP, an alkylating agent used for treating gastric cancer, ovarian cancer and diffuse malignant peritoneal mesothelioma, is the most common agent used during heat-enhanced IP chemotherapy (HIPC) (16). A combination of systemic and loco-regional chemotherapy may be effective in patients with carcinomatosis from gastric cancer, particularly in patients with small volumes of disease and symptomatic ascites (16).

The present study describes the use of bidirectional chemotherapy in the treatment of gastric cancer with PC, using newly developed response criteria regarding the treatment of malignant ascites. The present study also investigated the association between effusion response and patient survival.

\section{Materials and methods}

Patients. A total of 41 patients were enrolled in the present study, including $23(56.1 \%)$ males and 18 (43.9\%) females, with a median age of 42 (range, 31-69) years. The trial was conducted by the Department of Oncology, The Third Affiliated Hospital, Soochow University (Changzhou, China), between June 2010 and May 2014. Patients were eligible if they met the following criteria: Histopathological confirmation of gastric cancer; tumor cells in ascites, pathological findings of peritoneal metastasis or macroscopic PC diagnosed by laparoscopy; the absence of non-curative factors, including distant metastasis to the liver or lungs, with the exception of metastases to the peritoneum; Eastern Cooperative Oncology Group (ECOG) (17) performance status <2; age < 75 years; no prior treatment; adequate bone marrow function (leukocyte count $>3,000 / \mathrm{ml}$ and platelet count $>100,000 / \mathrm{ml}$ ); adequate liver function (serum bilirubin level $<1.5 \mathrm{mg} / \mathrm{dl}$ and serum transaminase levels $<2$ times the upper normal limit); adequate renal function (serum creatinine level $<1.5 \mathrm{mg} / \mathrm{dl}$ ); no other severe medical conditions, including symptomatic infectious disease, intestinal pneumonia, active hemorrhage/bleeding or obstructive bowel disease; not pregnant or lactating; provision of written informed consent in accordance with hospital regulations; and an expected survival time $>3$ months. Gastric cancer ascites was observed with a light microscope (BX51; Olympus Corporation, Tokyo, Japan) with x200 magnification.

The present study was approved by the Ethics Committee of Soochow University. All patients provided written informed consent regarding their involvement in the study, in accordance with institutional guidelines.

Bidirectional chemotherapy and HIPC. IP catheters, ARROWg+ and blue central venous catheters (Arrow International, Inc., Reading, PA, USA) were inserted under local anesthesia prior to therapy. On day 1 of every 3 -week cycle, heated $\left(43.0 \pm 0.5^{\circ} \mathrm{C}\right) \mathrm{CDDP}$ perfusion solution $(60 \mu \mathrm{g} / \mathrm{ml}$ saline for a dose of $75 \mathrm{mg} / \mathrm{m}^{2}$ ) was infused into the peritoneal cavity of all patients through an inflow tube using an automatic hyperthermia chemotherapy perfusion device (HGG-Z102: Hejia Medical Treatment Information Industry Co., Ltd., Zhuhai,China). Subsequent to perfusion, to allow the solution to distribute itself throughout the entire peritoneal surface, the tilt of the patient was changed at 15 min intervals during continuous perfusion over $2 \mathrm{~h}$ as follows: (1) level; (2) Trendelenburg + left tilt; (3) Trendelenburg + right tilt; (4) level; (5) reverse Trendelenburg + left tilt; and (6) reverse Trendelenburg + right tilt. Approximately $30 \mathrm{~min}$ was required to deliver a volume of 11. Additionally, all patients were infused intravenously for $1 \mathrm{~h}$ with docetaxel $\left(75 \mathrm{mg} / \mathrm{m}^{2}\right)$ on day 1 of the 3 week cycle.

Evaluation and determination of efficacy. Baseline evaluations included patient history, physical examination, measurement of the serum concentration of carcinoembryonic antigen (CEA) (18) and an examination of the abdomen using B-mode ultrasound within 1 week prior to therapy. CEA and a B-mode ultrasound inspection of the abdomen were repeated prior to each cycle of chemotherapy.

Malignant ascites is considered a non-evaluable lesion, since it is difficult to detect by conventional radiological examinations. The present study used a new response criterion to assess the effect of treatment of PC: B-mode ultrasound examination above the bladder using longitudinal sections prior to and following therapy. Complete response (CR) was defined as complete absence of effusion on B-mode ultrasound or CT scans; partial response (PR) as a $\geq 50 \%$ reduction in ascites depth on B-mode ultrasound; and non-PR (nPR) as a $<50 \%$ reduction or an increase in ascites depth. Patients were assessed by B-mode ultrasound following every second treatment cycle. The majority of patients received 6 cycles of HIPC and systemic chemotherapy.

Statistical analysis. Survival time was calculated from the initial date of treatment to the date of the most recent follow-up visit of the event of patient mortality, using the Kaplan-Meier method. Univariate analysis was performed using the log-rank test, and multivariate analysis was performed using a Cox proportional hazards model. $\mathrm{P}<0.05$ was considered to indicate a statistically significant difference. The primary study endpoint was overall survival (OS) rate, and the secondary endpoints were efficacy and serious adverse events, defined as severe local and/or systemic infection, intestinal occlusion or mortality associated with the procedure. The statistical analyses were performed using SPSS version 16.0. (SPSS Inc., Chicago, IL USA).

\section{Results}

Between June 2010 and May 2014, 41 patients were enrolled in the present study, including 17 with primary tumors and peritoneal dissemination, and 24 with peritoneal recurrence (Table I). The 41 patients consisted of $23(56.1 \%)$ males and 18 (43.9\%) females, with a median age of 42 (range, 31-69) years. All patients had an ECOG performance status score of 0 or 1 . Metastatic sites included the peritoneum (41/41; 100\%), lymph nodes $(13 / 41 ; 31.7 \%)$, liver $(12 / 41 ; 12.2 \%)$ and lungs $(2 / 41$; $4.9 \%)$, with 34 patients $(82.9 \%)$ having positive peritoneal cytology. Fig. 1 shows typical cancer cells in gastric cancer ascites. All patients presented with malignant ascites.

Fig. 2 shows a typical peritoneal effusion response imaged using B-mode ultrasound. Prior to treatment, numerous abdominal effusions were observed above the bladder (Fig. 2A). 
Table I. Characteristics of patients.

\begin{tabular}{lc}
\hline Variables & Patients, n $(\%)$ \\
\hline Age, years & $42.1 \pm 13.2^{\mathrm{a}}(31-69)$ \\
Sex & \\
Male & $23(56.1)$ \\
Female & $18(43.9)$ \\
ECOG performance status & \\
0 & $2(4.9)$ \\
1 & $13(31.7)$ \\
2 & $16(39.0)$ \\
Ascites & \\
No & $0(0.0)$ \\
Yes & $41(100.0)$ \\
Peritoneal lavage cytology & \\
Negative & $7(17.1)$ \\
Positive & $34(82.9)$ \\
Primary or recurrence & \\
Primary & $17(41.5)$ \\
Recurrence & $24(58.5)$ \\
Cycles of hyperthermic intraperitoneal & $2.2^{\mathrm{b}}(1-4)$ \\
perfusion chemotherapy & $41(100.0)$ \\
Total & \\
\hline
\end{tabular}

${ }^{a}$ Represents the mean $\pm \mathrm{SD}$, and ${ }^{\mathrm{b}}$ represents median; ECOG, Eastern Cooperative Oncology Group.

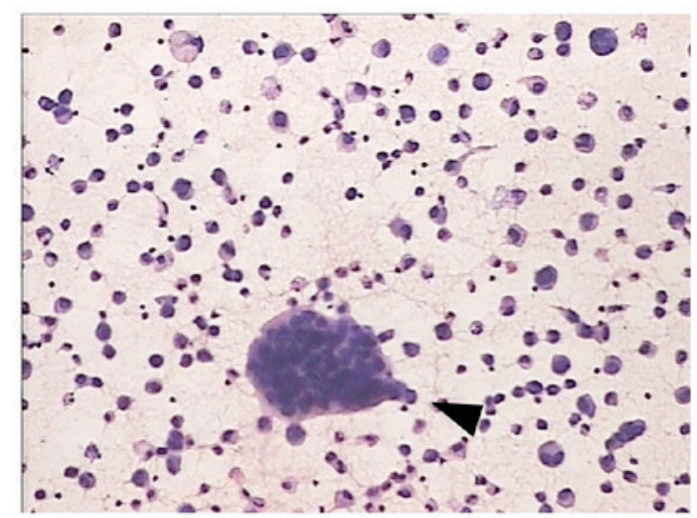

Figure 1. Cancer cells (indicated by arrow) in the ascites fluid of a patien with gastric cancer. Numerous cancer cells were present in the ascites prior to hyperthermic intraperitoneal perfusion chemotherapy (hematoxylin and eosin; magnification, x200).

Following two cycles of treatment, the peritoneal effusions had disappeared above the bladder (Fig. 2B), indicating that this patient had achieved CR. CT of another patient also showed a typical peritoneal effusion response during therapy (Fig. 3); this patient was also regarded as having achieved CR.

Table II shows the effects of therapy on peritoneal dissemination. The majority of patients showed clinical regression of ascites and associated symptoms. The peritoneal effusion response rate $(\mathrm{RR}=\mathrm{CR}+\mathrm{PR})$ was $73.2 \%$, including 7 patients (17.1\%) who achieved CR and 23 (56.1\%) who achieved PR,
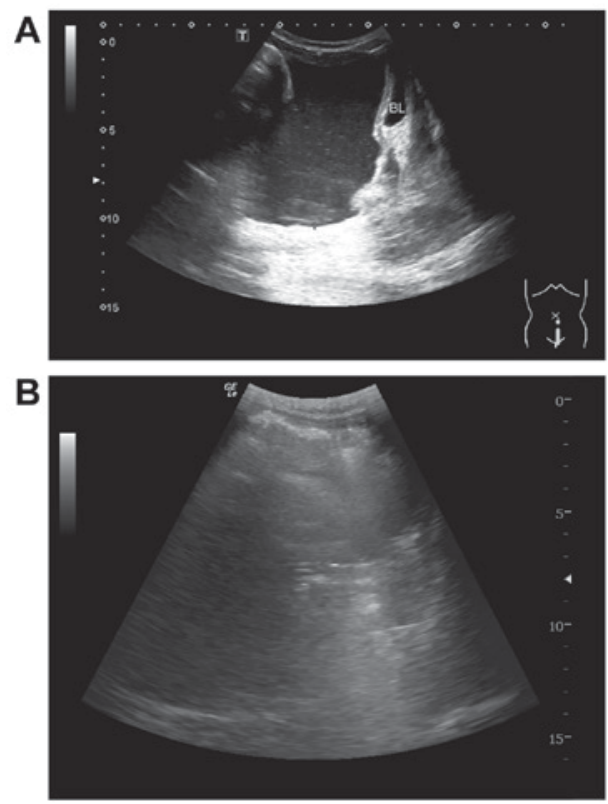

Figure 2. Longitudinal sections from B-mode ultrasound examinations above the bladder showing (A) symptomatic ascites prior to hyperthermic intraperitoneal perfusion chemotherapy and (B) disappearance of ascites following two cycles of therapy.

with the remaining 11 patients $(26.8 \%)$ achieving nPR. There were no mortalities associated with the procedure. At a median follow-up of 11.4 months, the mean survival time (MST) of all 41 patients enrolled in the present study was $8.5 \pm 0.7$ months. MSTs in CR, PR and nPR groups were 15.4 $\pm 5.6,9.1 \pm 0.9$ and $6.9 \pm 0.5$ months, respectively (Table II), with significant differences among the 3 groups $(\mathrm{P}<0.05$; Fig. 4). Kaplan-Meier analysis showed that the 1-year OS rate was $24.4 \%$ (Fig. 5).

\section{Discussion}

Peritoneal metastasis frequently occurs in patients with recurrent gastrointestinal malignancies (19). The most serious condition that may develop in peritoneal metastasis is PC, which has an extremely poor prognosis $(6,20,21)$. $\mathrm{PC}$ has been estimated to be responsible for $60 \%$ of all gastric cancer-associated mortalities, with peritoneal metastases in these patients considered terminal $(6,20,21)$. Therapy consists mainly of palliative chemotherapy; long-term survival is considered poor, since systemic chemotherapy agents are unlikely to reach cytotoxic concentrations in peritoneal nodules (22-27).

Although IP chemotherapy may deliver high-dose intensity treatment to the peritoneal cavity, drug penetration deep into the peritoneal surface is limited (28). Hyperthermia treatment may augment the penetration distance of anticancer drugs by up to $2,000 \mu \mathrm{m}$, as well as altering the permeability of tumor cell membranes to enhance uptake of chemotherapeutic drugs (28). In addition, the combination of hyperthermic treatment and chemotherapeutic drugs, including mitomycin $\mathrm{C}$, etoposide and CDDP have shown synergistic cytotoxicity towards cancer cells (29). Thus, combinations of systemic and loco-regional chemotherapy may be considered in patients with carcinomatosis from gastric cancer. These combinations may be particularly effective for patients with small volumes 
Table II. Effects of therapy on peritoneal dissemination.

\begin{tabular}{lcrr}
\hline Response & Patients, $\mathrm{n}(\%)$ & Mean survival time (months) & 95\% confidence interval \\
\hline $\begin{array}{l}\text { Disappeared (complete response) } \\
\begin{array}{l}\text { Decrease of ascites } \geq 50 \% \\
\text { (partial response) }\end{array}\end{array}$ & $7(17.07)$ & $15.4 \pm 5.6$ & $4.358-26.442$ \\
$\begin{array}{l}\text { Decrease of ascites }<50 \% \text { or increase } \\
\text { (non-partial response) }\end{array}$ & $11(26.83)$ & $9.1 \pm 0.9$ & $7.263-10.937$ \\
\begin{tabular}{l} 
Overall \\
\hline
\end{tabular} & $41(100.00)$ & $6.9 \pm 0.5$ & $5.929-7.871$ \\
\end{tabular}

$\mathrm{CR}$, complete response; $\mathrm{PR}$, partial response; $\mathrm{nPR}$, non-partial response.
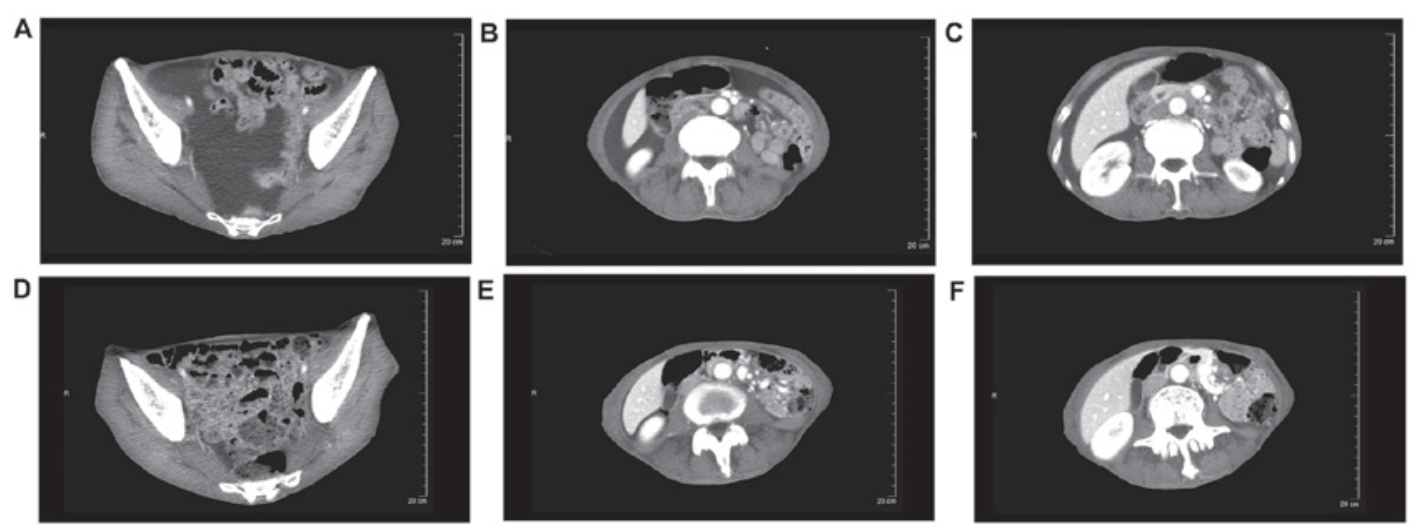

Figure 3. Typical peritoneal effusion response during therapy. CT images showing symptomatic ascites secondary to peritoneal metastasis prior to hyperthermic intraperitoneal perfusion chemotherapy: (A) pelvic region, (B) hepatorenal recess and (C) around the liver and kidney. Following 2 cycles of therapy, the patient was disease-free, as shown by the CT scans and was assessed as achieving complete response: (D) pelvic region, (E) hepatorenal recess, and (F) around the liver and kidney. CT, computed tomography.

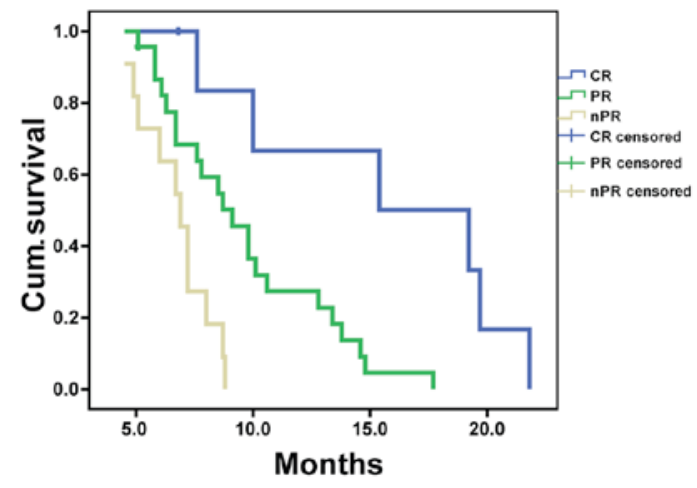

Figure 4. Kaplan-Meier overall survival curves for patients who achieved complete response, partial response and non-partial response. The mean survival time of the complete response group was $15.4 \pm 5.6$ months, which was the longest of the groups. There were significant differences among the 3 groups $(\mathrm{P}<0.05)$.

of disease and symptomatic ascites. A phase I/II trial revealed that IP docetaxel plusS-1 was safe and effective in patients with gastric cancer with PC (30).

The present study analyzed the effectiveness of a combination of systemic chemotherapy (intravenous docetaxel) and loco-regional chemotherapy (IP CDDP), with the two administered on day 1 of every 3 -week cycle. The present findings indicated that this therapeutic protocol was feasible and

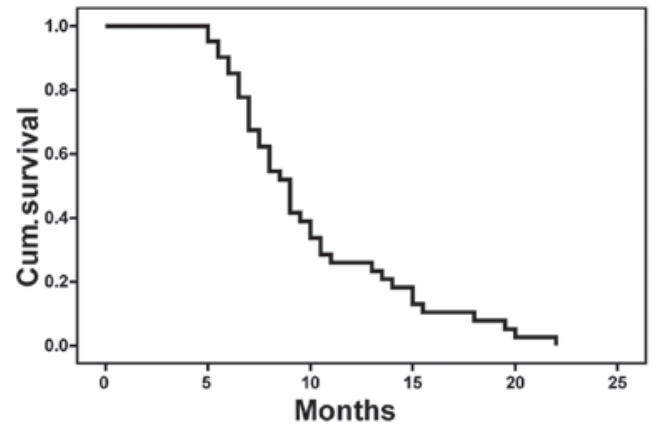

Figure 5. Kaplan-Meier analysis of overall survival for the 41 patients with peritoneal dissemination of gastric cancer enrolled in the present study. The median survival time was 8.6 months and the 1 -year survival rate was $24.39 \%$.

useful, and achieved satisfactory clinical outcomes. Another study reported that palliative treatment of ascites with IP instillation of mitoxantrone achieved an average decrease in ascites of $\geq 50 \%$ (31).

In daily clinical practice, CT was used to measure solid tumors, and the Response Evaluation Criteria in Solid Tumors 1.1 criteria (32) was used to determine the response to treatment. Malignant ascites were considered to be a non-evaluable lesion in the present study, since it is difficult to detect using conventional radiological examinations. Therefore, a simple 
method of estimating residual peritoneal ascites was established, and new response criteria were developed using B-mode ultrasound examination above the bladder in the longitudinal section. Therapeutic outcomes were categorized as CR, PR and $\mathrm{nPR}$, allowing its use in clinical settings.

The prognosis is poor for patients with macroscopic PC, which is responsible for $\sim 60 \%$ of all gastric cancer-associated mortalities $(33,34)$. Two prospective studies assessed outcomes in patients with PC from non-gynecologic malignancies: Gastric, colorectal and pancreatic cancer $(6,35)$. The presence of ascites was associated with poor survival of patients with gastric or pancreatic carcinoma. Analyses of factors prognostic for survival showed that differentiation of the primary tumor did not affect the prognosis of patients with PC. Mean and median OS times in these patients with PC from non-gynecologic malignancies were 6.0 and 3.1 months, respectively $(6,35)$.

In contrast to these earlier studies, which analyzed factors associated with survival in untreated patients, the patients in the present study received HIPC and intravenous chemotherapy. MST in all treated patients was $8.5 \pm 0.7$ months, being $15.4 \pm 5.6$ months in the CR group, $9.1 \pm 0.9$ months in the PR group and $6.9 \pm 0.5$ months in the nPR group, with significant differences between each pair of groups. These results provided evidence that the presence of ascites was associated with poor survival.

In conclusion, the present study indicated that B-mode ultrasound was effective and feasible in evaluating malignant ascites in patients with gastric cancer, as well as in determining response to treatment. The present study also demonstrated that the combination of HIPC and intravenous chemotherapy was effective and safe in patients with gastric cancer with malignant ascites. The overall response rate was $73.2 \%$, and patients who achieved CR exhibited significantly longer survival compared with those who achieved PR or nPR. Additional studies in larger populations are required to evaluate the efficacy and safety of this combination in carefully selected patients with gastric cancer with peritoneal metastases.

\section{References}

1. Ferlay J, Shin HR, Bray F, Forman D, Mathers C and Parkin DM: Estimates of worldwide burden of cancer in 2008: GLOBOCAN 2008. Int J Cancer 127: 2893-2917, 2010.

2. Parkin DM, Bray F, Ferlay J and Pisani P: Global cancer statistics, 2002. CA Cancer J Clin 55: 74-108, 2005.

3. Isobe Y, Nashimoto A, Akazawa K, Oda I, Hayashi K, Miyashiro I, Katai H, Tsujitani S, Kodera Y, Seto Y and Kaminishi M: Gastric cancer treatment in Japan: 2008 nnual report of the JGCA nationwide registry. Gastric Cancer 14: 301-316, 2011.

4. Koizumi W, Narahara H, Hara T, Takagane A, Akiya T, Takagi M, Miyashita K, Nishizaki T, Kobayashi O, Takiyama W, et al: S-1 plus cisplatin versus $\mathrm{S}-1$ alone for first-line treatment of advanced gastric cancer (SPIRITS trial): A phase III trial. Lancet Oncol 9: 215-221, 2008

5. Sakata Y, Ohtsu A, Horikoshi N, Sugimachi K, Mitachi Y and Taguchi T: Late phase II study of novel oral fluoropyrimidine anticancer drug S-1 (1 M tegafur-0.4 M gimestat-1 M otastat potassium) in advanced gastric cancer patients. Eur J Cancer 34: 1715-1720, 1998.

6. Sadeghi B, Arvieux C, Glehen O, Beaujard AC, Rivoire M, Baulieux J, Fontaumard E, Brachet A, Caillot JL, Faure JL, et al: Peritoneal carcinomatosis from non-gynecologic malignancies: Results of the EVOCAPE 1 multicentric prospective study. Cancer 88: 358-363, 2000.
7. McQuellon RP, Loggie BW, Fleming RA, Russell GB, Lehman AB and Rambo TD: Quality of life after intraperitoneal hyperthermic chemotherapy (IPHC) for peritoneal carcinomatosis. Eur J Surg Oncol 27: 65-73, 2001.

8. Garofalo A, Valle M, Garcia J and Sugarbaker PH: Laparoscopic intraperitoneal hyperthermic chemotherapy for palliation of debilitating malignant ascites. Eur J Surg Oncol 32: 682-685, 2006.

9. Glockzin G, Ghali N, Lang SA, Agha A, Schlitt HJ and Piso P: Peritoneal carcinomatosis. Surgical treatment, including hyperthermal intraperitoneal chemotherapy. Chirurg 78: 1100, 1102-1106, 1108-1110, 2007 (In German).

10. Roth AD, Fazio N, Stupp R, Falk S, Bernhard J, Saletti P, Köberle D, Borner MM, Rufibach K, Maibach R, et al: Docetaxel, cisplatin, and fluorouracil; docetaxel and cisplatin; and epirubicin, cisplatin, and fluorouracil as systemic treatment for advanced gastric carcinoma: A randomized phase II trial of the Swiss Group for Clinical Cancer Research. J Clin Oncol 25: 3217-3223, 2007

11. Dedrick RL, Myers CE, Bungay PM and DeVita VT Jr: Pharmacokinetic rationale for peritoneal drug administration in the treatment of ovarian cancer. Cancer Treat Rep 62: 1-11, 1978.

12. Markman M: Intraperitoneal antineoplastic drug delivery: Rationale and results. Lancet Oncol 4: 277-283, 2003.

13. Detroz B, Laurent S, Honoré P, Blaffart F, Limet R and Meurisse M: Rationale for hyperthermic intraperitoneal chemotherapy (HIPEC) in the treatment or prevention of peritoneal carcinomatosis. Acta Chir Belg 104: 377-383, 2004.

14. González-Moreno S, González-Bayón LA and Ortega-Pérez G: Hyperthermic intraperitoneal chemotherapy: Rationale and technique. World J Gastrointest Oncol 2: 68-75, 2010.

15. Van der Speeten K, Stuart OA and Sugarbaker PH: Using pharmacologic data to plan clinical treatments for patients with peritoneal surface malignancy. Curr Drug Discov Technol 6: 72-81, 2009.

16. Yan TD, Cao CQ and Munkholm-Larsen S: A pharmacological review on intraperitoneal chemotherapy for peritoneal malignancy. World J Gastrointest Oncol 2: 109-116, 2010.

17. Oken MM, Creech RH, Tormey DC, Horton J, Davis TE, McFadden ET and Carbone PP: Toxicity and response criteria of the Eastern Cooperative Oncology Group. Am J Clin Oncol 5: 649-655, 1982.

18. Tozzoli R, Basso SM, D'Aurizio F, Metus P and Lumachi F: Evaluation of predictive value of pleural CEA in patients with pleural effusions and histological findings: A prospective study and literature review. Clin Biochem 49: 1227-1231, 2016.

19. Yonemura Y, Endou Y, Sasaki T, Hirano M, Mizumoto A, Matsuda T, Takao N, Ichinose M, Miura M and Li Y: Surgical treatment for peritoneal carcinomatosis from gastric cancer. Eur J Surg Oncol 36: 1131-1138, 2010.

20. Yan TD, Black D, Savady R and Sugarbaker PH: Systematic review on the efficacy of cytoreductive surgery combined with perioperative intraperitoneal chemotherapy for peritoneal carcinomatosis from colorectal carcinoma. J Clin Oncol 24: 4011-4019, 2006.

21. Chua TC, Robertson G, Liauw W, Farrell R, Yan TD and Morris DL: Intraoperative hyperthermic intraperitoneal chemotherapy after cytoreductive surgery in ovarian cancer peritoneal carcinomatosis: Systematic review of current results. J Cancer Res Clin Oncol 135: 1637-1645, 2009.

22. Glehen O, Gilly FN, Arvieux C, Cotte E, Boutitie F, Mansvelt B, Bereder JM, Lorimier G, Quenet F and Elias D; Association Française de Chirurgie: Peritoneal carcinomatosis from gastric cancer: A multi-institutional study of 159 patients treated by cytoreductive surgery combined with perioperative intraperitoneal chemotherapy. Ann Surg Oncol 17: 2370-2377, 2010.

23. Kim JY and Bae HS: A controlled clinical study of serosa-invasive gastric carcinoma patients who underwent surgery plus intraperitoneal hyperthermo-chemo-perfusion (IHCP). Gastric Cancer 4: 27-33, 2001.

24. Glehen O, Schreiber V, Cotte E, Sayag-Beaujard AC, Osinsky D, Freyer G, François Y, Vignal J and Gilly FN: Cytoreductive surgery and intraperitoneal chemohyperthermia for peritoneal carcinomatosis arising from gastric cancer. Arch Surg 139: 20-26, 2004.

25. Van Cutsem E, Moiseyenko VM, Tjulandin S, Majlis A, Constenla M, Boni C, Rodrigues A, Fodor M, Chao Y, Voznyi E, et al: Phase III study of docetaxel and cisplatin plus fluorouracil compared with cisplatin and fluorouracil as first-line therapy for advanced gastric cancer: A report of the V325 Study Group. J Clin Oncol 24: 4991-4997, 2006. 
26. Scaringi S, Kianmanesh R, Sabate JM, Facchiano E, Jouet $P$, Coffin B, Parmentier G, Hay JM, Flamant Y and Msika S: Advanced gastric cancer with or without peritoneal carcinomatosis treated with hyperthermic intraperitoneal chemotherapy: A single western center experience. Eur J Surg Oncol 34: 1246-1252, 2008.

27. Yonemura Y,Endou Y,Shinbo M, Sasaki T, Hirano M, Mizumoto A, Matsuda T, Takao N, Ichinose M, Mizuno M, et al: Safety and efficacy of bidirectional chemotherapy for treatment of patients with peritoneal dissemination from gastric cancer: Selection for cytoreductive surgery. J Surg Oncol 100: 311-316, 2009.

28. Los G, van Vugt MJ and Pinedo HM: Response of peritoneal solid tumours after intraperitoneal chemohyperthermia treatment with cisplatin or carboplatin. Br J Cancer 69: 235-241, 1994.

29. Yonemura Y: Hyperthermo-chemotherapy for treatment of peritoneal dissemination. In: Peritoneal Dissemination. Yonemura Y (ed). Kanazawa: Maeda Shoten, pp237-260, 1998.

30. Fushida S, Kinoshita J, Kaji M, Hirono Y, Goda F, Yagi Y, Oyama K, Sudo Y, Watanabe Y and Fujimura T; Society for Study of Peritoneal Carcinomatosis in Gastric Cancer: Phase I/II study of intraperitoneal docetaxel plus S-1 for the gastric cancer patients with peritoneal carcinomatosis. Cancer Chemother Pharmacol 71: 1265-1272, 2013.
31. Link KH, Roitman M, Holtappels M, Runnebaum I, Urbanzyk H, Leder $\mathrm{G}$ and Staib L: Intraperitoneal chemotherapy with mitoxantrone in malignant ascites. Surg Oncol Clin N Am 12: 865-872, xvi-xvii, 2003.

32. Eisenhauer EA, Therasse P, Bogaerts J, Schwartz LH, Sargent D, Ford R, Dancey J, Arbuck S, Gwyther S, Mooney M, et al: New response evaluation criteria in solid tumours: Revised RECIST guideline (version 1.1). Eur J Cancer 45: 228-247, 2009.

33. Bando E, Yonemura Y, Takeshita Y, Taniguchi K, Yasui T, Yoshimitsu Y, Fushida S, Fujimura T, Nishimura G and Miwa K: Intraoperative lavage for cytological examination in 1,297 patients with gastric carcinoma. Am J Surg 178: 256-262, 1999.

34. Sugarbaker PH and Yonemura Y: Clinical pathway for the management of resectable gastric cancer with peritoneal seeding: Best palliation with a ray of hope for cure. Oncology 58: 96-107, 2000.

35. Chu DZ, Lang NP, Thompson C, Osteen PK and Westbrook KC: Peritoneal carcinomatosis in nongynecologic malignancy. A prospective study of prognostic factors. Cancer 63: 364-367, 1989. 